\title{
PERFIL DE HOMENS NA ATENÇÃO PRIMÁRIA À SAÚDE
}

\author{
F. P. SANTIAGO, P. R. F. SOUZA, F. C. A. MACHADO* e E. R. L. FERNANDES \\ Instituto Federal de Educação, Ciência e Tecnologia do Rio Grande de Norte \\ flavitamachado@yahoo.com.br ${ }^{*}$
}

Artigo submetido em julho/2015 e aceito em agosto/2015

DOI: $10.15628 /$ holos.2015.3214

\section{RESUMO}

Características próprias do universo masculino como o simbolismo da masculinidade podem dificultar práticas de autocuidado, bem como a procura por serviços de saúde, tornando este grupo mais vulnerável ao adoecimento. Com intuito de reverter este panorama, criou-se no Brasil a Política Nacional de Atenção Integral a Saúde do Homem (PNAISH). Assim, este estudo busca traçar o perfil de homens que acessaram serviços da atenção primária da Zona Norte de Natal/RN e verificar o conhecimento da PNAISH por este grupo. Para tanto, realizou-se um estudo seccional com 155 homens entre 20 e 59 anos que responderam a um questionário para caracterizá-los quanto: condição socioeconômica, hábitos, perfil de doença, conhecimento da PNAISH e percepção quanto ao serviço de saúde acessado. Assim foi constatado, dentre outros aspectos, que a maioria dos indivíduos eram pardos, tinham baixa escolaridade, renda entre 1 e 2 salários e desconheciam a PNAISH. Em relação ao serviço, $90,3 \%$ disseram ter resolvido sua demanda, porém apenas $55,4 \%$ consideraram a assistência satisfatória. Por conseguinte, homens que acessam a atenção primária são, em sua maioria, de baixa condição socioeconômica e desconhecedores da PNAISH. Assim, é necessário disseminar mais informações sobre esta política e adequar os serviços de saúde para atender as reais necessidades da população masculina.

PALAVRAS-CHAVE: Saúde do Homem; Gênero; Atenção Primária à Saúde; Política de Saúde

\section{MEN'S PROFILE IN PRIMARY HEALTH CARE}

\begin{abstract}
Specific Characteristics of the male's universe as the symbolism of masculinity may hamper self-care practices, as well the demand for health services, making the men more vulnerable to illness. To resolve this situation, it was created in Brazil the National Policy of Men's Integral Health Care (NPMIHC). Then, this study aims to delineate the profile of men who accessed primary health care services from the North Zone of Natal / RN and verify the knowledge of NPMIHC by this group. Therefore, it was performed a cross-sectional study with 155 men between 20 and 59 years old who answered a questionnaire to characterize them as: socioeconomic status, habits, disease profile, NPMIHC's
\end{abstract}

knowledge and perception of the health services accessed. Then it was found, among other things, that most individuals were brown, had lower education, income between 1 and 2 salaries and ignored the NPMIHC. Regarding the health service, $90.3 \%$ said they had solved their demand, but only $55.4 \%$ considered the service's assistance satisfactory. In this sense, men who access primary health care are, mostly, from low socioeconomic status and unaware of NPMIHC. Then, it's necessary to disseminate more information about this policy and adequate health services to meet the real needs of the male population.

KEYWORDS: Men's Health; Gender; Primary Health Care; Health Policy. 


\section{INTRODUÇÃO}

Desde os primórdios da vida, evidenciam-se as diferenças de gênero entre homens e mulheres que se formam e se modificam culturalmente de acordo com a sociedade e o tempo. Há tempos a sociedade é alicerçada em valores patriarcais que reforçam estigmas de superioridade, força, virilidade e invulnerabilidade ao homem e de sensibilidade e delicadeza a mulher. Assim, foi atribuído a mulher o cuidado afetivo do lar e da família, e ao homem o sustento da casa (MACIEL, 2009).

Este modelo hegemônico de masculinidade, adotado pela maior parte dos homens, contribui para a menor procura aos serviços de saúde e adoção de práticas de autocuidado, tornando-os mais vulneráveis às doenças, lesões e morte (KORIN, 2001). Desta forma, a busca ao serviço se dá quando já há uma morbidade perceptível da doença, ocasionando malefícios para si e um maior custo para o sistema de saúde. Isto porque quando buscam o serviço, geralmente optam ou, de fato, necessitam de uma assistência de complexidade secundária e terciária, distanciando-se da assistência primária (SCHWARZ et al., 2012).

Neste sentido, apesar de apresentar um maior índice de morbimortalidade, os homens procuram serviços de atenção primária à saúde em menor proporção que as mulheres (GOMES et al., 2008). Por isto, o Ministério da Saúde institui a Política Nacional de Atenção Integral à Saúde do Homem (PNAISH) por intermédio da portaria 1.944/2009. A PNAISH visa à redução da morbidade e mortalidade da população masculina do Brasil por meio da melhoria das condições de vida e enfrentamento racional dos fatores de risco à saúde. Desta forma, busca facilitar e estimular o acesso aos serviços de saúde em prol da equidade e integralidade (MINISTÉRIO DA SAÚDE, 2009).

Com a implantação da PNAISH, o Brasil passa a ser reconhecido como o segundo País das Américas a implantar uma política de saúde com ações específicas para o público masculino tendo o Canadá como o país pioneiro (VIEIRA et al., 2011). Diante disso, o presente estudo teve como objetivos traçar o perfil dos homens que buscaram assistência em unidades do Distrito Sanitário Norte I da cidade do Natal/RN e verificar o conhecimento sobre a existência da PNAISH. Tais objetivos justificam-se pela necessidade de identificar fatores que desencadeiam a procura por serviços de saúde pelos homens de modo a fornecer subsídios para planejar e desenvolver ações voltadas às demandas reais deste grupo e assim, estimular o acesso aos serviços. Quanto a PNAISH, é preciso investigar o conhecimento e o reconhecimento desta política pelo grupo alvo de suas ações, uma vez que já se transcorreram 03 anos desde sua instituição. Desta forma, é preciso analisar se a PNAISH está instituída ou não nas ações de serviços para, em caso contrário, traçar estratégias para sua efetiva instituição.

\section{MÉTODO}

Pesquisa exploratória, descritiva, de natureza quantitativa, realizada em 04 unidades básicas de saúde (UBS) da Zona Norte do Município de Natal/RN. Natal possui em sua área territorial quatro zonas administrativas, sendo a Zona Norte a maior, tanto em extensão territorial quanto em população. É a região que mais cresce no município, possuindo 83.152 homens na faixa etária de 20 a 59 anos de idade, num total de 303.543 habitantes (IBGE, 2010). 
As unidades disponibilizam serviços típicos da atenção básica à saúde como consultas médicas e de enfermagem, realização de curativos, tratamento odontológico, vacinas, fornecimento de medicações básicas e encaminhamentos para os demais níveis da atenção à saúde a depender da necessidade do usuário.

O estudo envolveu uma amostra de homens com idade entre 20 e 59 anos, usuários dos serviços das unidades no período de Março a Abril de 2013. Para estimativa do tamanho da amostra, foi utilizada como referência a quantidade de atendimentos nessas unidades no período de Março a Abril de 2012 ( $n=260$ ) e um nível de confiança de 95\%. Esses dados foram analisados com o auxílio do programa Sestatnet que determinou uma amostra de 155 homens.

Além da idade entre 20 e 59 anos, os homens que compuseram a amostra não poderiam apresentar doenças ou agravos que impossibilitassem o entendimento das perguntas, bem como do protocolo do estudo e deveriam se configurar como usuários dos serviços tendo comparecido às unidades de saúde em busca de assistência no período de Março a Abril de 2013 em que ocorreu a pesquisa.

Para possibilitar uma maior representatividade dos homens, procurou-se diversificar os dias e horários da coleta de dados. No entanto, o turno matutino foi privilegiado por disponibilizar uma maior oferta de serviços e ser apontado como o período de maior procura pelos usuários segundo os funcionários das unidades.

Para coleta de dados, utilizou-se um questionário semiestruturado, construído com base no instrumento de Albano, Basílio e Neves (2010). Assim, o questionário adaptado ao estudo vigente continha questões relativas à caracterização socioeconômica dos homens (idade, etnia, estado civil, escolaridade, ocupação, renda, no de filhos), hábitos (prática, tipo e frequência de atividade física; consumo vigente ou passado de álcool e tabaco; tempo em que parou o consumo de álcool e tabaco), perfil de doença (presença e tipo de patologia), perfil do serviço de saúde prestado na unidade (tipo do serviço procurado, opinião sobre o serviço utilizado, se houve resolução do problema que induziu à procura à UBS, tempo transcorrido desde o último acesso ao serviço), opinião sobre qual sexo busca mais os serviços da UBS e por fim, o questionamento sobre o conhecimento sobre a PNAISH.

Antes de iniciar a aplicação do questionário o participante recebeu instruções verbais sobre o objetivo e relevância da pesquisa e da garantia do anonimato. Após receber essas informações e concordar em participar do estudo, assinava um Termo de Consentimento Livre e Esclarecido. Todo o protocolo da pesquisa foi aprovado pelo Comitê de Ética em Pesquisa da Universidade Potiguar, com parecer de número 211.194 e CAAE de número 11507013.7.0000.5296.

Os dados coletados foram processados no software gratuito GNU PSPP (Program for Statistical Analysis of Sampled Data) para obtenção de suas frequências absolutas e relativas, bem como medidas de tendência central.

\section{RESULTADOS}

Os homens que buscaram assistência nas unidades de saúde tinham em média 39,27 anos de idade $( \pm 12,44)$, havendo uma distribuição equilibrada entre adultos jovens $(49,03 \%)$ e adultos 
na faixa etária de 41 a 51 anos (50,97\%). Esses homens eram em sua maioria pardos, casados ou em união estável e tinham de 1 a 3 filhos.

No que se refere à escolaridade, $35,49 \%(n=55)$ dos participantes concluíram o ensino médio e apenas $2,58 \%(n=4)$ concluíram o ensino superior. Isto foi refletido na renda mensal onde $72,26 \%(n=112)$ apresentam renda mensal de 1 a 2 salários mínimos. A ocupação mais frequente foi a categoria empregado e autônomo ( $n=114 ; 73,54 \%)$.

Os dados referentes a esta caracterização sociodemográfica estão representados nas figuras 1 e 2 a seguir.

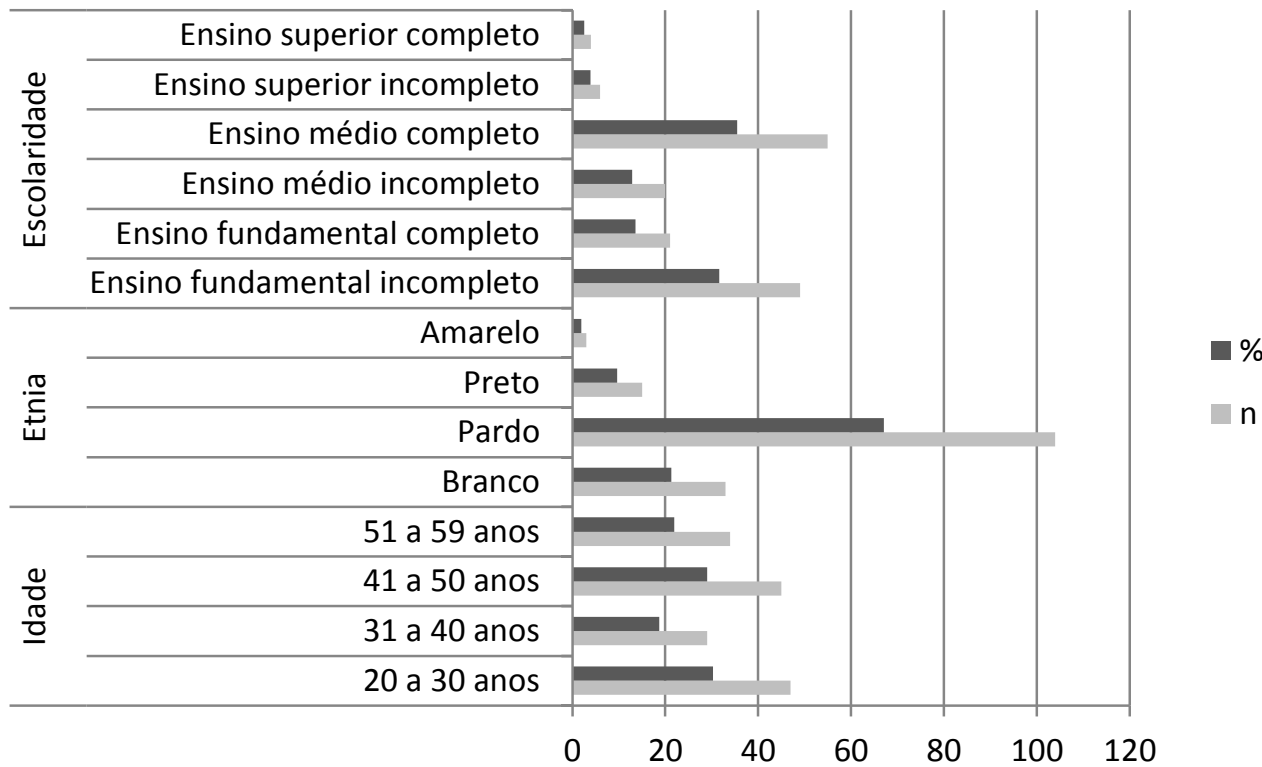

Figura 1: Caracterização dos homens ( $n=155)$ quanto à idade, etnia e escolaridade. Fonte: Dados primários coletados nas UBS envolvidas neste estudo.

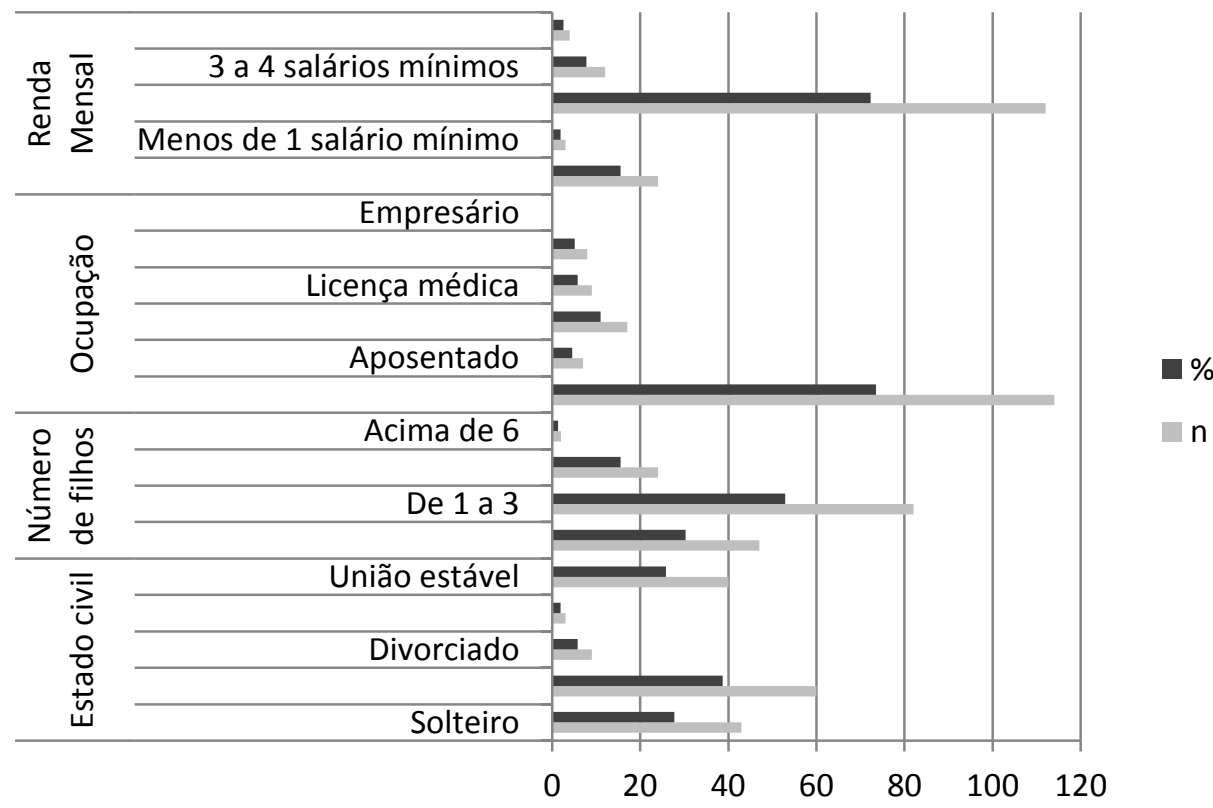

Figura 2: Caracterização dos homens ( $n=155)$ quanto ao estado civil, $n$ o de filhos, ocupação e renda mensal. Fonte: Dados primários coletados nas UBS envolvidas neste estudo. 
Em relação aos hábitos, a grande parte dos entrevistados são etilistas ou já foram etilistas. Porém, o tabagismo não foi um hábito expressivo visto que o percentual de ex-tabagistas também foi baixo (Tabela 1). Esses ex-tabagistas pararam com o hábito há uma média de 10,30 anos ( $\pm 7,32$ anos), enquanto os ex-etilistas há 5,02 anos ( $\pm 5,12$ anos). Quanto à atividade física, $67,74 \%$ ( $n=105)$ não pratica e daqueles que praticam, a principal modalidade é a de exercício aeróbico $(n=33 ; 66 \%$ ) realizada de 3 a 6 dias na semana (média $=3,76$; d.p. $\pm 0,94$ ).

Tabela 1: Perfil dos homens participantes da pesquisa relacionado aos hábitos de vida.

\begin{tabular}{|c|c|c|c|}
\hline Variável & Categoria & $\mathbf{N}$ & $\%$ \\
\hline \multirow{3}{*}{$\begin{array}{l}\text { Etilismo } \\
(\mathrm{N}=155)\end{array}$} & Etilistas & 70 & 45,00 \\
\hline & Não etilistas & 55 & 36,00 \\
\hline & Ex-etilistas & 30 & 19,00 \\
\hline \multirow{3}{*}{$\begin{array}{c}\text { Tabagismo } \\
(\mathrm{N}=155)\end{array}$} & Tabagistas & 14 & 9,03 \\
\hline & Não Tabagistas & 128 & 82,58 \\
\hline & Ex-tabagistas & 13 & 8,39 \\
\hline $\begin{array}{c}\text { Prática de atividade } \\
\text { física }\end{array}$ & Sim & 50 & 32,26 \\
\hline$(\mathrm{N}=155)$ & Não & 105 & 67,74 \\
\hline $\begin{array}{c}\text { Tipo de atividade } \\
\text { Física }\end{array}$ & Exercício aeróbico & 33 & 66,00 \\
\hline$(N=50)$ & Exercício anaeróbico & 17 & 34,00 \\
\hline
\end{tabular}

Quanto ao perfil de doenças, $60,00 \%(n=93)$ dos homens declararam não serem portadores de doenças crônicas. Dentre as patologias crônicas autoreferidas pelos participantes, houve preponderância da hipertensão arterial associada ou não com outras patologias (ver tabela 2).

Tabela 2: Perfil dos homens participantes da pesquisa relacionado a doenças crônicas.

\begin{tabular}{cccc}
\hline Variável & Categoria & $\mathbf{N}$ & $\%$ \\
\hline & Hipertensão Arterial & 18 & 29,03 \\
Perfil de Doenças auto & Diabetes Mellitus & 06 & 9,67 \\
referidas pelos homens & Cardiopatia & 02 & 3,23 \\
$(\mathbf{N = 6 2 )}$ & Diabetes e outros & 03 & 4,84 \\
& Hipertensão e outros & 09 & 14,52 \\
& Diabetes e Hipertensão & 04 & 6,45 \\
& Diabetes, hipertensão e outros & 03 & 4,84 \\
& Diabetes, hipertensão, cardiopatia e outros & 01 & 1,61 \\
& Hipertensão e cardiopatia & 03 & 4,84 \\
& Outros & 13 & 20,97 \\
\hline
\end{tabular}

Fonte: Dados primários coletados nas UBS envolvidas neste estudo.

Por fim, em relação ao serviço, este foi bem avaliado, já que 90,3\% ( $n=140$ ) dos entrevistados disseram ter resolvido sua demanda, havendo preponderância de uma opinião positiva da assistência. O motivo principal que levaram os homens a procura de assistência na UBS foi a realização de consultas médicas (ver figura 3).

Quanto à opinião sobre quem mais utiliza os serviços de saúde, $100 \%$ dos entrevistados disseram ser as mulheres. Quando questionados sobre a PNAISH, apenas 15,5\% ( $n=24)$ disseram conhecê-la. 


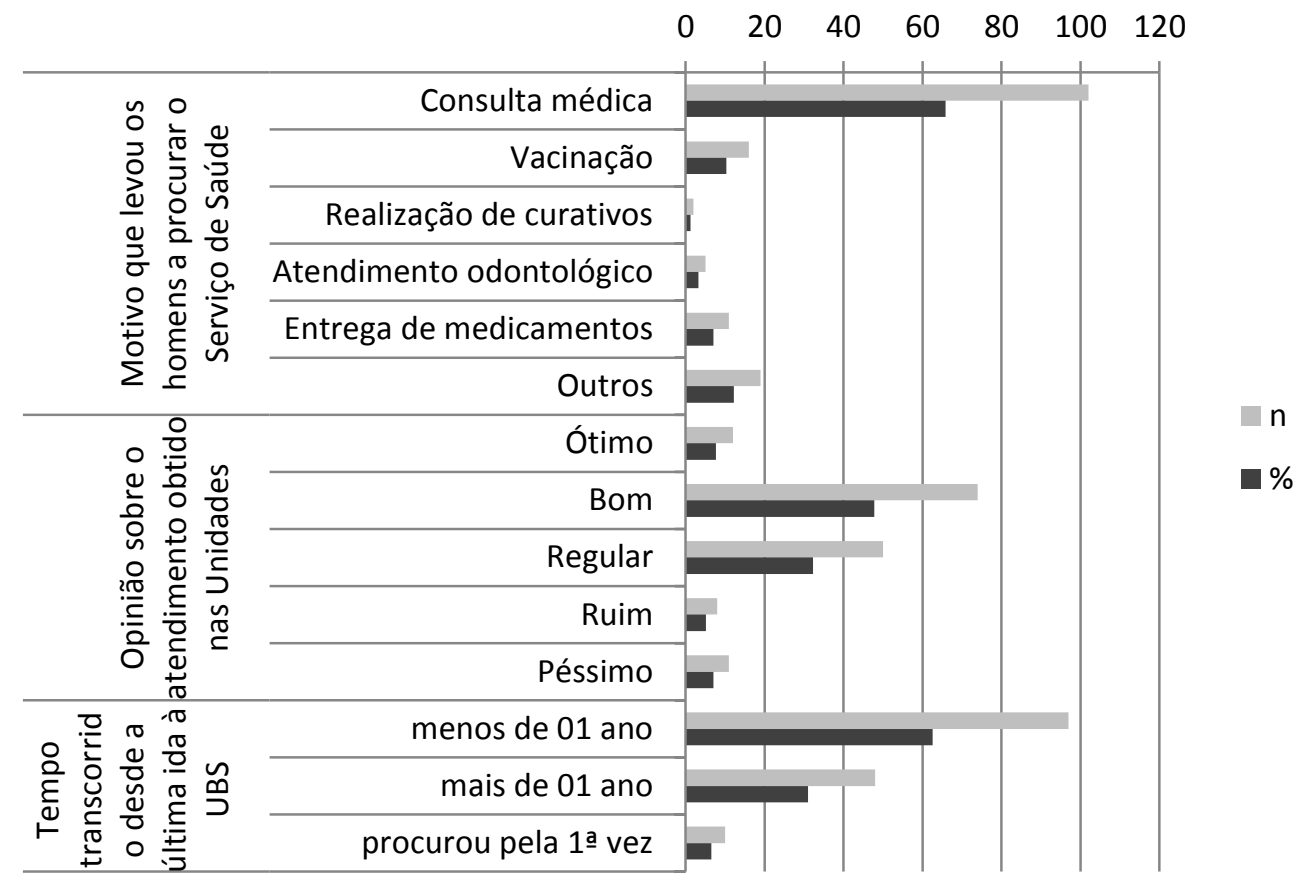

Figura 3: Caracterização dos serviços de saúde prestados nas Unidades Básicas aos homens participantes do estudo.

Fonte: Dados primários coletados nas UBS envolvidas neste estudo.

\section{DISCUSSÃO}

Os resultados mostram que dos homens entrevistados, esses eram em sua maioria, adultos jovens, pardos, empregados com renda de 1 a 2 salários mínimos, casados ou em união estável.

Quanto aos hábitos deste grupo, 64\% dos homens consomem ou já consumiram bebidas alcoólicas. De fato, o consumo de álcool é comum no universo masculino e representa uma tendência de crescimento na sociedade contemporânea. Neste sentido, a OMS enfatiza que tal tendência também ocorre no Brasil e que os homens apresentam uma maior prevalência de consumo regular de álcool em relação às mulheres (BORTOLUZZI et al., 2010). Isso os torna mais vulneráveis às doenças cardiovasculares, neoplasias, absenteísmo - falta ao trabalho, aposentadorias precoces, acidentes de trabalho e de transporte, episódios de violência agressões, homicídios, suicídios e elevada frequência de ocupação de leitos hospitalares (MASCARENHAS et al., 2009).

Em vista disso, percebe-se a necessidade de políticas públicas de promoção à saúde que busquem conscientizar esta parcela da população quanto aos malefícios relacionados ao consumo abusivo de álcool. Uma vez que, as doenças que tem como fator de risco o consumo de álcool estão no ranking das principais causas de óbito da população independente do sexo (BRASIL, 2008).

Em relação ao consumo do tabaco, ao contrário do estudo de Barros et al (2011), que aponta para uma elevada frequência do tabagismo entre os homens, o presente estudo apontou que a maior parte dos homens entrevistados não faziam uso do tabaco. Isto vem a contribuir para a saúde dos mesmos, já que em conformidade com o INCA (2011), o tabagismo contribui 
para o desenvolvimento de diferentes tipos de câncer: pulmão, boca, faringe, laringe, esôfago, estômago, pâncreas, bexiga, rim, colo do útero e leucemia mielóide aguda, doença pulmonar obstrutiva crônica (DPOC), doença coronariana, hipertensão arterial e acidente vascular encefálico.

Ainda com relação ao tabagismo, devemos enfatizar que há uma intensa campanha pública veiculada em rádio e televisão informando verbalmente e visualmente a respeito da morbimortalidade associada a este hábito. Isto favorece uma redução do índice de tabagismo, em vista do poder dos meios de comunicação em massa em formar opiniões e potencializar mudanças de hábitos. Neste sentido, campanhas evidenciando a morbimortalidade associada ao etilismo não são comuns, à exceção das que demonstram a associação entre mortalidade e consumo de álcool ao dirigir. Assim, percebe-se a necessidade de difundir maiores informações sobre os malefícios do álcool à nossa qualidade de vida tanto em termos das doenças associadas ao seu consumo como do prejuízo social que acarreta.

Quanto à prática de atividade física, observou-se não ser um hábito frequente entre os participantes da pesquisa. Considerando-se que a prática de exercícios físicos acarreta uma melhor qualidade de vida e contribui para a redução de riscos de morte prematura e de morbimortalidade (FEITOSA, PONTES, 2011) percebe-se uma demanda para ações de promoção de saúde da população.

Dentre os indivíduos que disseram realizar atividade física, a caminhada foi o exercício mais frequentemente apontado. Isto, provavelmente, está relacionado à grande divulgação sobre os benefícios da caminhada nos meios de comunicação em massa e em campanhas de prevenção às cardiopatias. Além disso, este exercício pode ser realizado gratuitamente nos espaços públicos e em horários escolhidos pelos indivíduos.

Analisando-se a congruência dos dados, a maior frequência da caminhada era esperada posto que a hipertensão arterial associada ou não a outras doenças foi destaque entre as doenças autoreferidas. Em verdade, a Hipertensão Arterial é considerada como um grande problema de saúde pública por se constituir em um dos maiores fatores de risco de mortalidade por doenças cardiovasculares, apresentando uma elevada prevalência na população masculina (SBC, 2010). Contudo, as doenças cardiovasculares apresentam uma significativa frequência em ambos os sexos (COSTA, THULER, 2012; SANTOS et al., 2013). Assim, o sedentarismo pode configurar-se como um potencial fator de risco para a população masculina.

No entanto, apesar do sedentarismo, houve uma preponderância de homens que relataram não possuir doenças crônicas. Isto pode ter ocorrido pela faixa etária de indivíduos incluídos no estudo, 20 a 59 anos, e também pelo baixo percentual de tabagistas.

Em relação ao perfil dos serviços prestados nas unidades, as consultas médicas destacaram-se como o motivo desencadeador da busca a assistência nas unidades de saúde. Isto está em consonância com Gomes et al (2011) que afirma que a procura pelos serviços de saúde na Atenção Primária por parte dos homens se dá quando estes apresentam alguma queixa. Ou seja, os homens remetem a assistência o tratamento da doença e de suas sequelas, mas não a prevenção e promoção à saúde. Tal fato, possivelmente, está relacionado ao modelo de masculinidade adotado por grande parte dos homens, no qual estes são vistos como seres fortes, invulneráveis ao adoecimento, contribuindo para ausência do autocuidado por parte destes e 
retardando ao máximo a procura por serviços de saúde na Atenção Primária (GOMES et al., 2008).

Neste sentido, o estudo mostrou uma unanimidade quanto à opinião sobre quem mais procura os serviços de saúde, as mulheres. Isto vai ao encontro dos estudos de Couto et al (2010), no qual foi constatado uma predominância feminina na maioria das atividades, assim como em quase todo espaço físico das unidades de saúde. As mulheres eram maioria nas consultas, nas salas de espera, nas filas, nos grupos e nas áreas de circulação (COUTO et al., 2010).

Os homens associam os cuidados com a saúde ao universo feminino, por considerarem as mulheres como seres mais frágeis, mais vulneráveis às doenças, necessitando de uma maior atenção à saúde. Além disso, elas teriam uma maior disponibilidade de tempo na busca por serviços de saúde (SCHRAIBER et al., 2010).

Quanto ao conhecimento acerca da Política Nacional de Atenção Integral a Saúde do Homem (PNAISH), revela-se claramente que apenas uma minoria dos participantes sabia sobre a existência da PNAISH. Essa minoria relatou ter obtido algumas informações sobre a PNAISH por meio da mídia, que realiza a divulgação de algumas ações como a prevenção do câncer de próstata.

Leal, Figueiredo e Silva (2012) apontam em seus estudos que a PNAISH ainda é pouco divulgada, havendo uma necessidade de se investir na divulgação dessa política tanto para população como para os profissionais da saúde. Ressalta-se ainda que, a criação da política foi um passo importante rumo ao aprimoramento do atendimento à saúde do público masculino, contudo, ainda não foi possível observar mudanças efetivas no Sistema Único de Saúde (SUS). Ainda é notória nas unidades de saúde a pouca oferta de programas ou atividades direcionadas especificamente para saúde dos homens, indicando existir uma dificuldade de interação entre as necessidades de saúde da população masculina e a organização das práticas de saúde das unidades de Atenção Primária (SILVA et al., 2012).

Apesar dos participantes do estudo terem considerado o atendimento das unidades de saúde como bom, os mesmos relataram lacunas na prestação da assistência por parte das unidades, tais como: dificuldade para o agendamento de consultas, difícil acesso, mau atendimento por parte dos funcionários, destacando-se nas falas dos depoentes a falta de médicos especialistas, falta de medicamentos, entre outros.

Vale salientar que a opinião do usuário quanto ao serviço prestado pelas unidades de saúde é um importante elemento de avaliação da qualidade da assistência oferecida, contribuindo assim para criação de propostas que venham melhorar a realidade dos serviços ofertados.

\section{CONCLUSÃO}

Homens pardos, casados ou com união estável, com baixa escolaridade, baixa renda, não portadores de doenças crônicas, etilistas e sedentários constituem o perfil de usuários masculinos da Atenção Primária. Assim, há uma demanda de ações de promoção à saúde em vista de expressiva quantidade de indivíduos com hábitos insalubres. Contudo, o baixo 
percentual de tabagistas/ex-tabagistas é um dado positivo em prol da redução dos riscos à saúde.

Esses homens não procuram os serviços para fins preventivos, já que as consultas médicas, preponderantemente curativas, constituem o principal motivo da procura. Neste ponto, poderíamos inferir que a criação da Política Nacional de Atenção Integral a Saúde do Homem (PNAISH) não influenciou ou motivou a procura em vista do alto percentual de desconhecimento desta política pelos entrevistados.

Isto nos leva a concluir que essa política não é bem divulgada para a população masculina e que há uma deficiência nas Unidades de Saúde da Atenção Primária em relação à oferta de programas de saúde voltados para a assistência quanto à promoção da saúde e prevenção de doenças que venham acometer a população masculina.

Conclui-se ser necessária uma maior divulgação da PNAISH e que esta seja, de fato, implantada nas Unidades de Saúde, possibilitando o desenvolvimento de programas que contribuam para a oferta de uma assistência integral e humanizada, considerando a singularidade do universo masculino. Assim, a PNAISH poderá, potencialmente, ser conhecida e reconhecida pela população, tornando-se instituída no âmbito do SUS.

\section{REFERÊNCIAS BIBLIOGRÁFICAS}

1. ALBANO, B.R.; BASÍLIO, M.C.; NEVES, J.B. Desafios para inclusão dos homens nos serviços de Atenção Primária. Revista Enfermagem Integrada, v.3, n.2, p. 554-563, 2010.

2. BARROS, A.J.D.; CASCAES, A.M.; WEHRMEISTER, F.C.; MESA, J.M.; MENEZES, A.M.B. Tabagismo no Brasil: desigualdades regionais e prevalência segundo características ocupacionais. Ciência \& Saúde Coletiva, v.16, n.9, p.3707-3716, 2011.

3. BORTOLUZZI, M. C.; TRAEBERT, J.; LOGUERCIO, A.; KEHRIG, R. T. Prevalência e perfil dos usuários de álcool de população adulta em cidade do sul do Brasil. Ciência \& Saúde Coletiva, v.15, n.3,p.679-685, 2010.

4. BRASIL. Ministério da Saúde, Instituto Nacional de Câncer (INCA), Organização PanAmericana de Saúde (OPAS). Pesquisa especial de tabagismo - PETab: relatório Brasil. Rio de Janeiro: INCA; 2011. Disponível em:< http://bvsms.saude.gov.br/bvs/publicacoes/pesquisa _especial_tabagismo_petab.pdf >. Acesso em: 10 abr. 2013.

5. BRASIL. Ministério da Saúde. Diagnóstico da saúde da população masculina. Brasília; 2009. Disponível em: <http://portal.saude.gov.br/portal/aplicacoes/noticias/default.cfm?pg=dsp DetalheNoticia\&id_area=124\&CO_NOTICIA=10491>. Acesso em: 07 set. 2012.

6. BRASIL. Ministério da Saúde, Secretaria de Atenção à Saúde, Departamento de Ações Programáticas Estratégicas. Política Nacional de Atenção Integral à Saúde do Homem (princípios e diretrizes). Brasília: MS; 2008. Disponível em: <http://portal.saude.gov.br/ portal/arquivos/pdf/politica_nacional_homem.pdf>. Acesso em: 18 set. 2012.

7. COSTA, L. C.; THULER, L.C.S. Fatores associados ao risco para doenças não transmissíveis em adultos brasileiros: estudo transversal de base populacional. Rev. bras. estud. popul., v. 29, n. 1, p. 133-145, jun. 2012.

8. COUTO, M. T.; PINHEIRO, T. F.; VALENÇA, O.; MACHIN, R.; SILVA, G. S. N.; GOMES, R.; SCHRAIBER, L. B.; FIGUEIREDO, W. S. et al . O homem na atenção primária à saúde: 
discutindo (in) visibilidade a partir da perspectiva de gênero. Interface- Comunicação, Saúde, Educação, v.14, n.3, p.257-270, abr./jun.2010.

9. FEITOSA, R.C.L.; PONTES, E. R.J.C. Levantamento dos hábitos de vida e fatores associados à ocorrência de câncer de tabagistas do município de Sidrolândia (MS, Brasil). Ciência \& Saúde Coletiva, v.16, n.2, p.605-613, 2011.

10. GOMES, R.; MOREIRA, M.C.N.; NASCIMENTO, E.F.; REBELLO, L.E.F.S.; COUTO, M.T.; SCHRAIBER, L.B. Os homens não vêm! Ausência e/ou invisibilidade masculina na atenção primária. Ciência \& Saúde Coletiva, v.16, n.1, p.983-992, 2011.

11. GOMES, R.; NASCIMENTO, E.F.; REBELLO, L.E.F.S.; ARAÚJO, F.C. As arranhaduras da masculinidade: uma discussão sobre o toque retal como medida de prevenção do câncer prostático. Ciência \& Saúde Coletiva, v.13, n.6, p.1975-1984, nov./dez.2008.

12. IBGE - Instituto Brasileiro de Geografia e Estatística. Sidra, censo. Brasil, 2010. Disponível em: < http:/www.sidra.lbge.gov.br>. Acesso em: 11 out. 2011.

13. KORIN D. Novas perspectivas de gênero em saúde. Adolescência Latinoamericana, v.2, n.2, p.67-79, mar.2001.

14. LEAL, A.F.; FIGUEIREDO, W.S.; SILVA, G.S.N. O percurso da Política Nacional de Atenção Integral à Saúde do Homem (PNAHISH), desde a sua formulação até sua implementação nos serviços públicos locais de atenção à saúde. Ciência \& Saúde Coletiva, v.17, n.10, p.26072616, 2012.

15. MACIEL, P.S.O. O homem na Estratégia de Saúde da Família. Natal: UFRN, 2009. 81 p. Dissertação (Mestrado) - Programa de pós-graduação em enfermagem, Universidade Federal do rio Grande do Norte, Natal, 2009.

16. MASCARENHAS, M.D.M.; MALTA, D.C.; SILVA, M.M.A.; CARVALHO, C.G.; MONTEIRO, R.A.; MORAIS NETO, O.L. Consumo de álcool entre vítimas de acidentes e violências atendidas em serviços de emergência no Brasil, 2006 e 2007. Ciência \& Saúde Coletiva, v.14, n.5, p.17891796, 2009.

17. SANTOS, V.P.; ALVES, C.A.S.; LOPES, C.F.; ARAÚJO FILHO, J.S. Diferenças entre os gêneros em pacientes com isquemia crítica por doença arterial obstrutiva periférica. J Vasc Bras, v. 12, n. 4, p.278-283, out./ dez.2013.

18. SCHWARZ, E.; GOMES, R.; COUTO, M.T.; MOURA, E.C.; CARVALHO, A.S.; SILVA, S.F.C. Política de Saúde do Homem. Revista de Saúde Pública, v.46, n.1, p.108-116, dez. 2012.

19. SCHRAIBER, L.B.; FIGUEIREDO, W.S.; GOMES, R.; COUTO, M.T.; PINHEIRO, T.F.; MACHIN, R.; SILVA, G.S.N.; VALENÇA, O. Necessidades de saúde e masculinidades: atenção primária no cuidado aos homens. Cadernos de Saúde Pública, Rio de Janeiro, v.26, n.5, p.961-970, maio 2010.

20. SILVA, P.A.S.; FURTADO, M.S.; GUILHON, A.B.; SOUZA, N.V.D.O.; DAVID, H.M.S.L. A saúde do homem na visão dos enfermeiros de uma unidade básica de saúde. Escola Anna Nery Revista de Enfermagem, v.16, n.3, p.561-568, jul./set. 2012.

21. SOCIEDADE BRASILEIRA DE CARDIOLOGIA. VI Diretrizes Brasileiras de Hipertensão. Rio de janeiro: SBC; 2010. Disponível em: <http://www.cardiol.br> Acesso em: 10 abr. 2013.

22. VIEIRA, L.C.S.; FIGUEIREDO, M.L.F.; SALES, R.L.U.B.; LOPES, W.M.P.S.; AVELINO, F.V.D. A política nacional de saúde do homem: uma reflexão sobre a questão de gênero. Enfermagem em Foco, v.2, n.4, p.215-217, 2011. 